\title{
A Design of Anthropomorphic Hand based on Human Finger Anatomy
}

\author{
Zixun $\mathrm{He}^{1}$, Yousun Kang ${ }^{2}$, Duk Shin ${ }^{3, *}$ \\ ${ }^{1}$ Department of Human Centered Science and Biomedical Engineering, Tokyo Institute of Technology, Yokohama, 226-8503, Japan \\ ${ }^{2}$ Department of Applied Computer Science, Tokyo Polytechnic University, Kanagawa, 243-0297, Japan \\ ${ }^{3}$ Department of Electronics and Mechatronics, Tokyo Polytechnic University, Kanagawa, 243-0297, Japan
}

\begin{tabular}{l} 
A R T I C L E I N F O \\
\hline Article history: \\
Received: 21 April, 2021 \\
Accepted: 09 August, 2021 \\
Online: 28 August, 2021 \\
\hline Keywords: \\
Human hand biomechanics \\
Prosthetic hand \\
EMG signals \\
Quality of life
\end{tabular}

\section{Introduction}

This paper is an extended paper of our work initially presented in International Symposium on Community-centric Systems (CcS 2020) [1]. The human hand is an important tool for us to interact with and perceive the physical environment. Upper limbs loss is one of the most disabling diseases that a person may experience, which can severely affect amputees Activities of daily living (ADLs) and working abilities. In the United States alone, there are approximately 50,000 people who have lost upper limbs, and it is predicted that the numbers of upper limb loss will increase over time [2]. According to the investigation [3], different amputees may have different requirements for prostheses. Besides performance of prosthetics devices, a high level of personification and simplified operating system are also required. Prosthetic developers need to consider these different requirements, which bring challenges to the development of prosthetic hands.

In order to improve the acceptability of the prosthesis [4,5], various artificial hand designs and models have been proposed. Commercially available advanced prosthetic hands, such as

*Corresponding Author: Duk Shin, 1583, Iiyama, Atsugi, Kanagawa, 243-0297,

Japan, Email: d.shin@eng.t-kougei.ac.jp

www.astesj.com

https://dx.doi.org/10.25046/aj060448
Michelangelo hand (by Otto Bock), are easy to use and can help amputee grip the objects. However, the price of commercially available prosthetics is very expensive. It is difficult for people who have lost upper limbs to afford this financial burden. In recent

years, with the development of new manufacturing technologies such as fused deposition modeling (FDM) 3D printers, low-cost and easy-to-make open-source prostheses have also been designed $[6,7]$.

Most research groups aim to replicate the movement mechanism of human hands in a mechanized design, improving the flexibility and performance of the prosthetics. In the past two decades, the research community has developed some novel artificial hands [8-10]. Lee et al. proposed the design of a 9- DOF bio-mimetic robot hand. This hand has four under-actuated fingers, each of finger is equipped with a tactile sensor and driven by two linear actuators coupled (together) [11]. In order to further reduce the weight of the prosthetic hand, Mohammadi et al. used soft materials and developed a lightweight soft robotic prosthetic hand that has synergy-based motion and cable-driven actuation system. In addition, successfully performed three different grips with high dynamic power $(21.5 \mathrm{~N})$ and fast finger bending speed. Although these artificial hands have excellent grip and performance, they 
have not yet reached the level of human dexterity. The reason for that is these designs adopt the structure of the human body in the mechanized way, the incomplete human hand-like motion mechanism limited to the motion performance of the manipulator [12].

In [13], the author developed the anatomically corrected tested (ACT) hand by replicating the bone structures of the fingers, which is the first robotic hand developed based on human hand interpretation. Although its mechanism is still based on hinges and universal joints, it still exhibits a more flexible grip than previous manipulators. In [14], the author designed a robot hand through high bionics, and a flexor mechanism for an elastic pulley mechanism to realize the joint movement of human fingers. These studies of highly bionic anthropomorphic robotic hands have showed the possibility of replicating the structure of the human hand on the prosthesis to make the movements more dexterity.

Electromyography signal (EMG) is a signal that can reflect the user's movement intention. In recent years, it has been widely used to control prostheses or robots. Although this kind of EMG-based control is widely used for pattern recognition and rehabilitation, the nature of EMG signal is random or non-stationary and will be affected by nearby muscles that generate additional noise [15]. Therefore, in order to control the prosthetic hand accurately, it is necessary to extract the features related to the selected action patterns, and then use support vector machine (SVM), linear discriminant analysis (LDA), Artificial neural network (ANN) and other machine learning to classify the action. The correct selection of features is critical to classification performance. However, because of different electrode placement schemes, number of channels, and EMG signal preprocessing methods in different studies, it is difficult to compare clearly which features can significantly improve the accuracy of classification [16]. Deep learning methods have recently been used in the classification of EMG signals and have shown strong performance [17]. CôtéAllard et al. use Convolutional Neural Networks (CNN) to classify the actions of 7 forelimbs with an accuracy of $97.9 \%$ [18]. It can be said that in a laboratory environment, such a classifier with high computational cost and powerful performance is satisfactory, but it is an important practical consideration to ensure the effectiveness while reducing the computational burden when building the system. In addition, reducing the number of electrodes to improve comfort also needs to be considered for the prosthetic hand system. Tavakoli et al. use only 2 EMG channels to classify four types of gestures, and the classification accuracy rate exceeds to $90 \%$ [19]. In daily life, we use hand movements to grasp objects or interact with the environment [20,21]. Therefore, by defining the minimum number of daily grasping patterns, the number of electrodes can be reduced, and the simplicity of the system can be maintained.

In this article, we designed an anthropomorphic prosthetic hand based on anatomical structure. The four-finger and thumb model of the prosthetic hand is modeled based on laser scanned hand bone data on cadaveric. This can reduce the rejection of the amputee to the prosthesis after the artificial skin is worn on the prosthetic hand, and the movement angle of each finger will not exceed the movable range of the original finger. Incorporate soft tissue structures such as ligaments, tendons and tendon sheaths that have a significant impact on the flexibility of the fingers at each joint to replicate the movement characteristics of the human hand. Then he conducted a grasping experiment on the prosthetic hand to test the performance of our prosthesis in daily life. In order to control the prosthetic hand using Electromyogram (EMG) signals, an EMG control system is designed for the artificial hand by an artificial neural network (ANN). The EMG control system is used to control the four main hand positions used in daily life: Power grasps, Precision grasps, Lateral grasps and relax state [21].

\section{Development of prosthetics}

\subsection{Hand structures and the Bones Models}

We designed highly anthropomorphic prosthetic hand based on the anatomy. First, the skeletal structure of the human finger movement mechanism is mainly determined by the metacarpal and phalangeal bones. The human finger comprises the distal, middle, proximal phalanges and metacarpals. These four bones make up the distal interphalangeal (DIP), proximal interphalangeal (PIP) and metacarpophalangeal (MCP) joints of the hand. The thumb finger has only two phalanges, so it has only one interphalangeal joint. Every finger joint has soft tissues called ligaments [22].

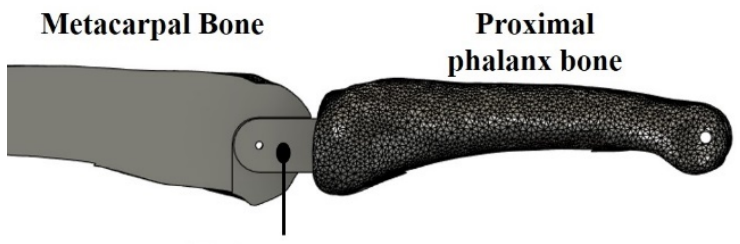

Link structure

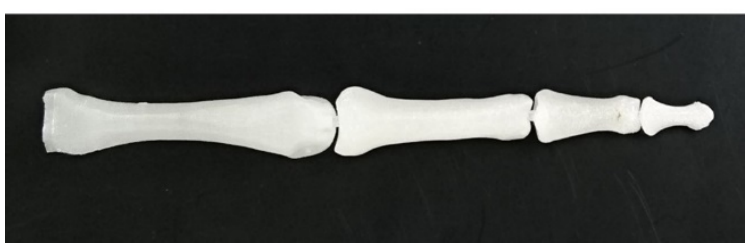

Figure 1: Designed Finger model

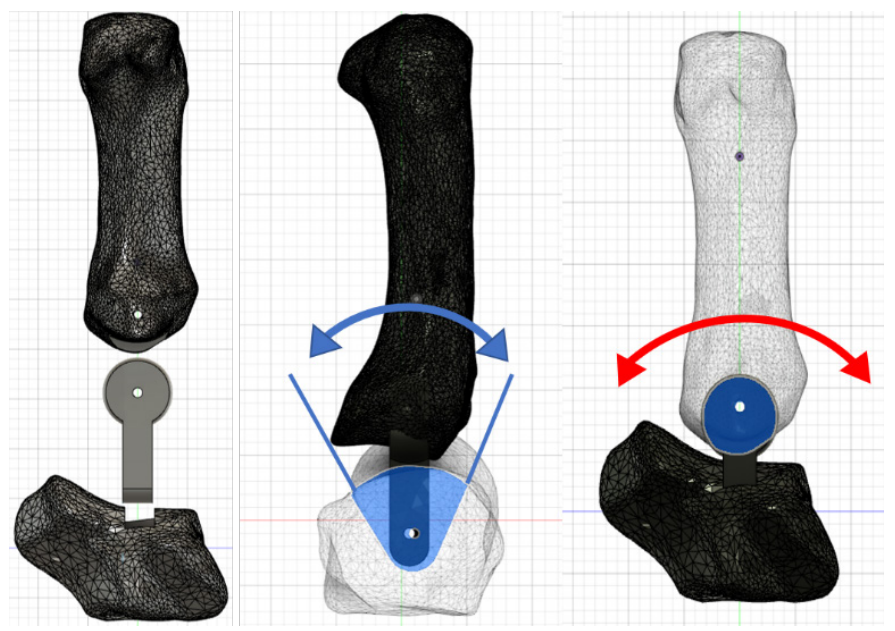

Figure 2: Proposed thumb design

We used laser-scanned bone data as the basic model of the prosthesis [23]. Since the size of the prosthetic hand skin in the market vendor is larger than the bone model of this hand, we adjusted the size of the model. In order to improve the efficiency of power transmission, a link structure is designed between each joint as shown as Figure 1. 
Table 1: Finger Flexion degree [deg]

\begin{tabular}{|c|c|c|}
\hline DIP Joint & PIP Joint & MCP Joint \\
\hline $0-77$ & $0-105$ & $0-90$ \\
\hline
\end{tabular}

The range of motion of the finger joints will vary between different fingers and different people, depending on the bone shape, tendons and muscle structure characteristics of the hand. By referring to the previous research on joint structure [24], in this research, their range of motion is uniformly designed, as shown in Table 1.

Although the thumb comprises only two bones, it has great freedom of movement. The reason is attributed to the carpometacarpal (CMC) joint composed of the thumb metacarpal bone and trapezium bone. The trapezium bone is shaped like a saddle used for horse riding, allowing the thumb to move in a larger range. The movement of the thumb is caused by sliding the metacarpal bone of the thumb along the trapezium bone at the CMC joint back and forth (extension / flexion), left and right (abduction / adduction) or both at the same time [25]. The following method is used to reproduce the thumb structure to ensure correct thumb movement. In order to reproduce the adduction and abduction functions of the thumb, the connecting rod structure on the metacarpal model additionally uses a rotary joint, as shown in Figure 2. And a groove is designed on the trapezium bone to achieve flexion and extension movement.

\subsection{Design of Joint Ligament and Tendon}

Ligaments are a type of fibrous connective tissue that connects bone to bone. These tissues can be found in all joints. The range of motion of each finger joint is limited by the length of the ligament. There are collateral ligaments on both sides of the hand bones, which are attached to the volar plates. Cartilage structures called volar plates are inserted on both sides of the bone joints. Volar plates and ligaments form a joint pack to prevent joint overextend and enhance joint stability. There are two sets of tendons in the human hand to stretch and bend the fingers. They are the extensor and flexor tendons. The flexor tendons extend from the forearm and, finally, branches become the flexor digitorum superficialis (FDS) tendon and the flexor digitorum profundus (FDP) tendon. The FDS tendon is fixed to the intermediate phalange bones of the PIP joint. The FDP tendon is inserted into the bottom of the palm of the distal phalanges. When the finger is bent, the PIP joint attached to the FDS tendon will bend first. As the movement progresses, the FDP tendon will play a role in bending the DIP joint. These two flexor tendons are firmly attached to the phalanx through the tendon sheath. The extensor tendons are combined with extensor hood that regulate joint extension and flexion. Extensor hood is a complex mesh structure that wraps the phalanx directly from the back of the hand. Its structure is shown in Figure 5 (a) [26]. The first layer of the dorsal aponeurosis of the extensor hood is inserted into the base of the DIP joint and divided into two small tendons at the PIP joint. The second layer of the dorsal aponeurosis of the extensor hood is located at the bottom of the PIP joint.

According to the ligament, the replicated model of the structure of the volar plate is shown in Figure 3. The volar plate is made of High Elasticity Rubber Plate. Anchor the rubber volar plate to each joint with screws. The ligament is made of rubber thread, and the natural length of the ligament is adjusted when inserted into the volar plate and both sides of the hand bone, so that the fingers of the artificial hand can be naturally bent like a human finger when they are naturally relaxed. Thus, the prosthetic hand will not be kept in a straight and rigid motion, and the prosthetic hand will be more personified.
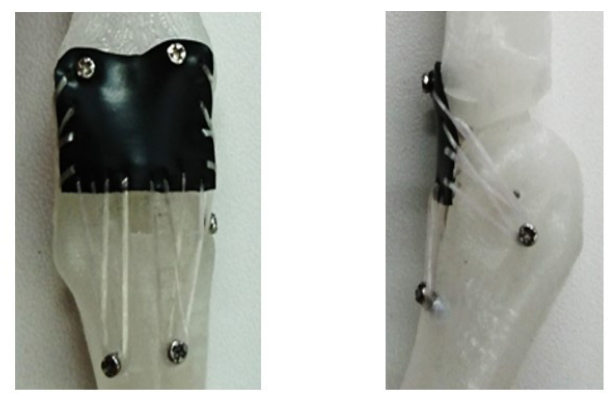

Figure 3: Structure in anatomy and reproduced model

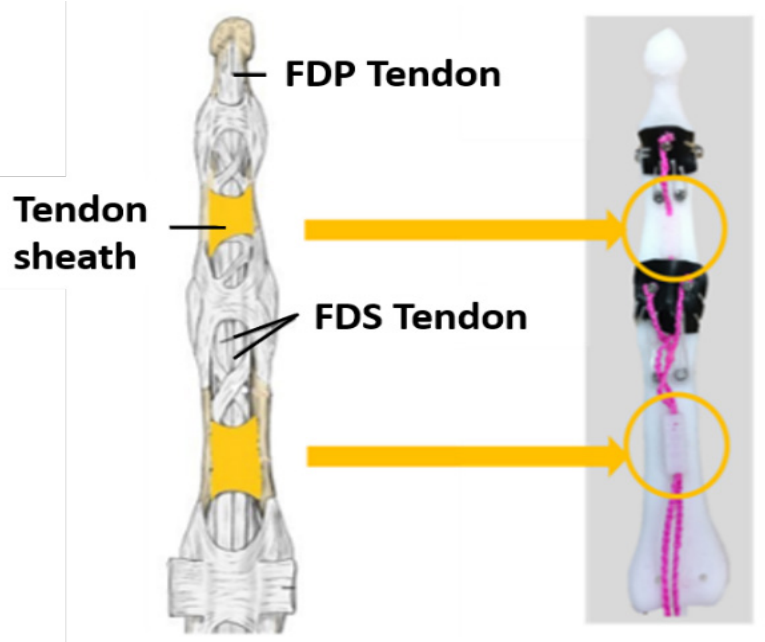

Figure 4: Tendon and Tendon sheaths

The flexor and extensor tendons composed of FDS tendons and FDP tendons are made of $0.33 \mathrm{~mm}$ polyethylene wire (Figure 4), with chief strength ( $250 \mathrm{~N}$ breaking strength) and good flexibility. The tendons are woven to prevent the volar plate and tendon sheath from being worn out because of the long-term movement of the tendons.

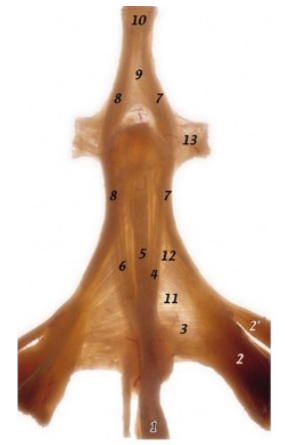

(a)

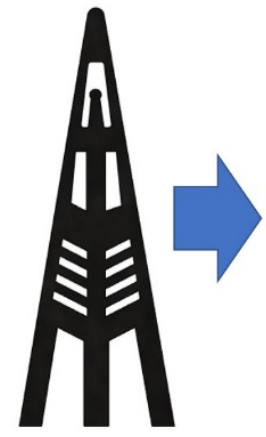

(b)

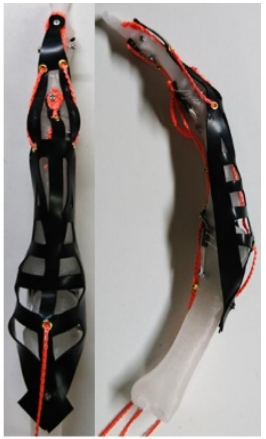

Figure 5: Hood parts; (a) Extensor hood Structure [26] (b) Reproduced extensor hood sheath 


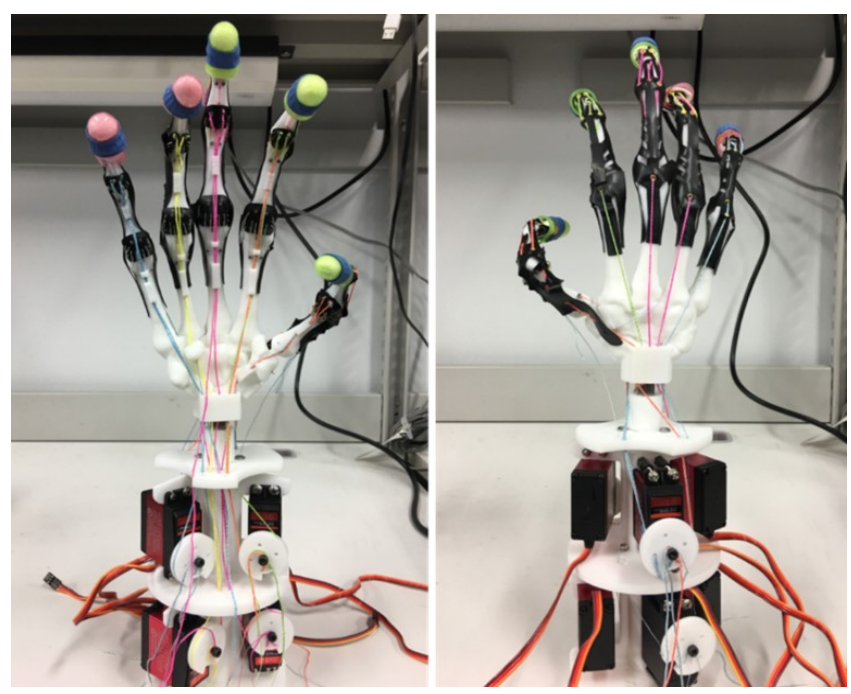

Figure 6: Configuration of the prosthesis

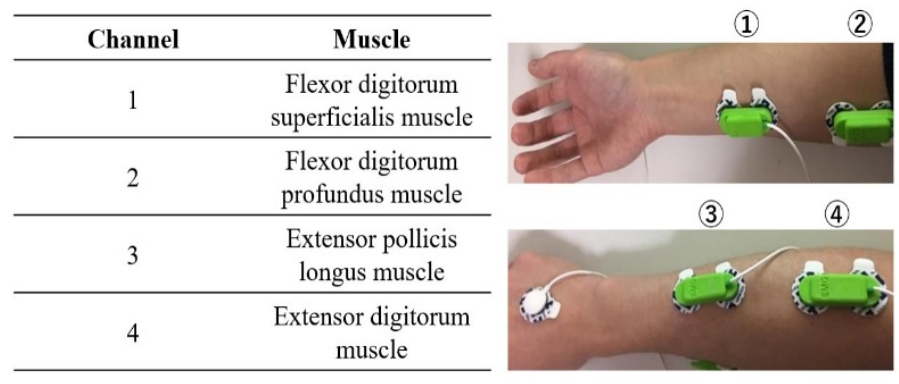

Figure 7: Location of the EMG electrodes



Figure 8: Pre-Processing of EMG signal

Table 2: The specifications of DS3218 Servo

\begin{tabular}{|c|c|}
\hline Dimensions & $40 \times 20 \times 40.5 \mathrm{~mm}$ \\
\hline Weight & $60 \mathrm{~g}$ \\
\hline Stall Torque & $21.5 \mathrm{~kg} \cdot \mathrm{cm}(6.0 \mathrm{~V})$ \\
\hline
\end{tabular}

The extensor tendon has a central fiber bundle that reaches the base of the intermediate phalanges while two outer fiber bundles are anchored at the base of the distal phalanx. We simplified the structure of the extensor hood and made the extensor hood using a highly elastic rubber sheet to reproduce the function of the extensor hood to adjust joint extending or bending. Figure 5 shows the finger after all components have been assembled.

\subsection{Configuration of the prosthesis and Control Method}

After all fingers are assembled, combined them with the wrist part. The complete prosthesis is shown in Figure 6. The tendon of the finger is fixed on the pulley, and the pulley is connected to the servo. By pulling the tendon with the servo motor, the finger joint bent or extended. Six Servo motors (Ds servo DS3218) are used to control the prosthetic hand. The servo specifications are shown in Table 2. Considering Thumb has 2 sets of movement, we use 2 servos to control Adduction or Abduction, and Flexion or Extension of the thumb, respectively. Other fingers are each controlled by one servo. In order to use the intuitive operation method to control the prosthetic hand in the grasping experiment, an operating system was developed using the right-hand glove part of Perception Neuron (Noitom, Beijing, China). Perception Neuron's joint obtain the movement of the finger detected in threedimensional coordinates. The servo is controlled by the DIP joint angle detected by the sensor neuron. Calculate the bending angle of the finger according to formula (1).

$$
\theta=180-\arccos \frac{z}{\sqrt{x^{2}+y^{2}+z^{2}}}
$$

Here, $\mathrm{x}, \mathrm{y}$, and $\mathrm{z}$ are three-dimensional coordinates detected from the sensory neuron of the DIP joint. The calculated angle data will be sent to Arduino UNO to control the servo.

\subsection{EMG signals Recording and Processing}

The author showed that increasing the number of electrodes does not always improve the accuracy of gesture classification [27]. In addition, problems such as using many electrodes or the long time for classification can also make amputee annoying.

Based on these basic demands, we decided on the number of electrodes and the classification time. The connection points of the EMG sensor and the surface muscles selected to measure the EMG signal are shown in Figure 7. These muscles control the four movements (power grasp, precision grasp, lateral grasp and relax) used for classification. The reason for choosing these 4 kinds of motions is because power grasps, precision grasps, Lateral grasps are most used in activities of daily living and user will also need a relax mode for an inactive position.

We gained EMG signals using the BITalino EMG sensor. By using MATLAB's Bitalino'Toolbox, the EMG signal is recorded on MATLAB at a sampling rate of $1000 \mathrm{~Hz}$ [28]. Before calculating the features of the EMG signal, we rectified and filtered the four EMG signal channels. All preprocessing are performed using MATLAB. A second-order Butterworth low-pass filter with a cutoff frequency of $5 \mathrm{~Hz}$ is used to filter the EMG signal to reduce the noise of the EMG signal [29]. Figure 8 shows the raw EMG signal and filtered EMG signal. During training and experiment, all features were analyzed using a sliding time 
window of $200(\mathrm{~ms})$ with an overlap of $100(\mathrm{~ms})$. The calculation processing delay is about $300(\mathrm{~ms})$. The control is quasi real-time.

\subsection{Feature Extraction}

Three types of signal features: time-domain features, frequency-domain features, and time-frequency features are usually used for EMG classification. In order to improve the accuracy of classification, two or more features are usually combined. We tested 8 EMG signal features proposed by Phinyomark et al. in the previous work to select features [30]. These features are: Integrated EMG (IEMG), Mean Absolute Value (MAV), Simple Square Integral (SSI), Variance of EMG (VAR), Root Mean Square (RMS), Waveform Length (WL), Mean Frequency (MNF) and Median Frequency (MDF). Among them, we found that the combination of the three features of RMS, $\mathrm{WL}$ and $\mathrm{MNF}(\mathrm{o})$ showed better results. These results are consistent with previous research results, root mean square (RMS) and wavelength (WL) can get good classification results and require less computational cost [31]. Compared with time-domain features such as RMS and WL, frequency-domain features require more computational costs. In [32], the author reported that the frequency-domain features are better than the time-domain features when predicting the angle and flexion of the thumb. Therefore, this study uses a combination of two time domain features RMS, WL and a frequency-domain features Mean Frequency (MNF) for classification.

The WL, RMS, MNF are defined as follows:

$$
\begin{gathered}
W L=\sum_{n=1}^{n-1}\left|x_{n+1}-x_{n}\right| \\
R M S=\sqrt{\frac{1}{N} \sum_{i=1}^{N} x_{i}^{2}} \\
M N F=\sum^{M} F P / \sum^{M} P
\end{gathered}
$$

here, $x_{n}$ denotes the $n^{\text {th }}$ of EMG signal, $N$ is the length of signals. $F$ is the frequency, $P$ is the Power spectrum.

Table 3: The list of objects used in the Experiment

\begin{tabular}{|c|c|c|c|}
\hline Number & Name & Weight (g) & Size(cm) \\
\hline 1 & TV Remote & 75 & length 13 width 5 thickness 3 \\
\hline 2 & Pill & 1 & diameter 1 \\
\hline 3 & Telephone & 137 & length 17 width 5 thickness 2 \\
\hline 4 & Bottle & 40 & length 13 width 5 thickness 3 \\
\hline 5 & Glasses & 38 & length 15 width 3 thickness 3 \\
\hline 6 & Spoon & 5 & length 15 width 3 thickness 2 \\
\hline 7 & Phone & 190 & length 16 width 7 thickness 1 \\
\hline 8 & Tooth Paste & 238 & length 13 width 5 thickness 3 \\
\hline 9 & Envelope & 14 & length 23 width 12 \\
\hline
\end{tabular}

\begin{tabular}{|c|c|c|c|}
\hline 10 & $\begin{array}{c}\text { Drink } \\
(500 \mathrm{ml})\end{array}$ & 534 & length 21 width 7 thickness 7 \\
\hline 11 & Bowl & 57 & length 11 width 11 thickness 5 \\
\hline 12 & Key & 9 & length 7 width 3 thickness 0.5 \\
\hline 13 & Pen & 15 & length 14 width 1 thickness 1 \\
\hline 14 & Paper box & 73 & length 18 width 11 thickness 8 \\
\hline 15 & Bill & 1 & length 15 width 7 \\
\hline 16 & Straw & 2 & length 10 width 0.5 \\
\hline 17 & Coin & 5 & diameter 2.5 \\
\hline 18 & Bag & 897 & length 40 width 25 thickness 15 \\
\hline 19 & DVD & 16 & diameter 11 \\
\hline 20 & Small Ball & 12 & diameter 3 \\
\hline
\end{tabular}

\section{Evaluation Experiment}

\subsection{Grasping experiment}

In order to evaluate the applicability of the proposed prosthesis to daily life, we used the items in the list of items that amyotrophic lateral sclerosis (ALS) patients are difficult to pick up in daily life for experiments [33]. We tested the feasibility of the prosthetic hand grasped and pick up easily some items as shown in Table 3. The gripping and dexterity must be needed the equivalent strength of strings driven like an actual hand. We have selected 20 items in the item list above. Among them, 19 items can be easily found in the laboratory. In addition, a school bag of about $1 \mathrm{~kg}$ was selected as the object to observe the performance of the artificial hand against heavy objects in Appendices. In grasping an item, if the prosthesis can grip for the object over 5 seconds, it will be recorded as a success, otherwise, it will be recorded as a failure.

\subsection{Classification experiment}

In order to classify four gestures, we used MATLAB's neural network toolbox to build a three-layer artificial neural network (ANN) as a classifier. This ANN consists of twelve (four channels $\mathrm{x}$ three features) nodes of input layers, one hidden layer composed of twelve nodes, and four nodes output layers. The activation functions of the middle layer and the output layer use hyperbolic tangent functions. The training method uses a learning algorithm called Levenberg-Marquardt backpropagation (LMBP). LMBP is a general non-linear least-squares optimization method, its major advantage lies in the speed of convergence. Before starting the Classification Experiment, we will train their own ANN for each subject in advance. The dataset used for training ANN contains 10 sets of data for each motion, and each set of data contains 5 seconds of EMG signal. In order to prevent over fitting, the training of ANN will stop if Mean Square Error (MSE) $\leqq 0.001$. Before performing the Classification experiment, use 10-fold crossvalidation on the collected dataset to test the accuracy of the classifier as shown as Figure 8 . For each subject's dataset, $80 \%$ of the data is used to train the classifier, and the remaining $20 \%$ are used to validate the performance of the classifier. Figure 9 shows the average accuracy of the four motions of 4 subjects after using 10 -fold cross-validation. The highest accuracy rate is $94.7 \%$ (Subject4), the lowest accuracy rate is $91.4 \%$ (Subject2). 


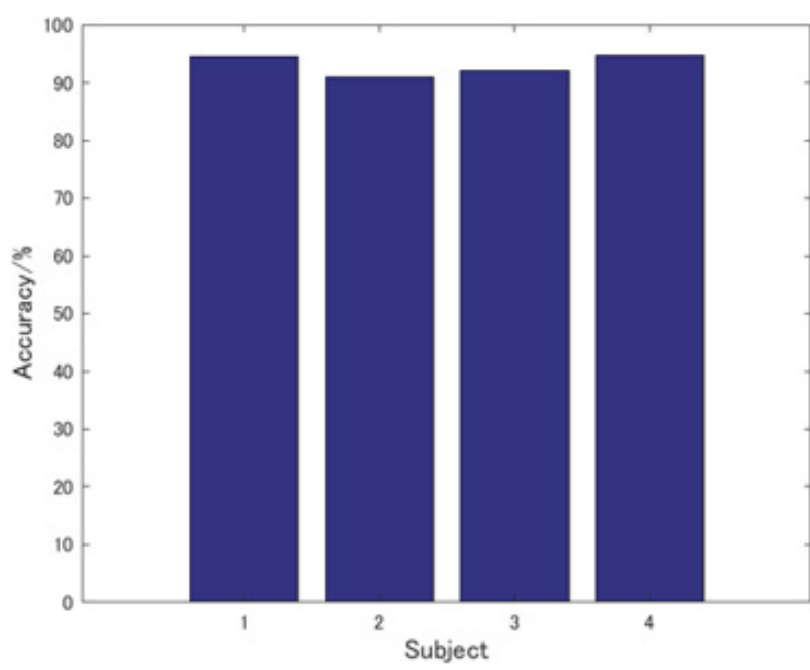

Figure 9: 10-fold cross validation results

\section{Results}

\subsection{Result of Grasping experiment}

In the experiment, our prosthetic hand successfully grasped selected 20 objects (Figure 10). Different from the traditional ON/OFF clip-shaped prosthetic hand or the prosthetic hand that grasps in a fixed posture, the proposed anthropomorphic design allows our prosthetic hand to grasp objects in the suitable grasping posture.

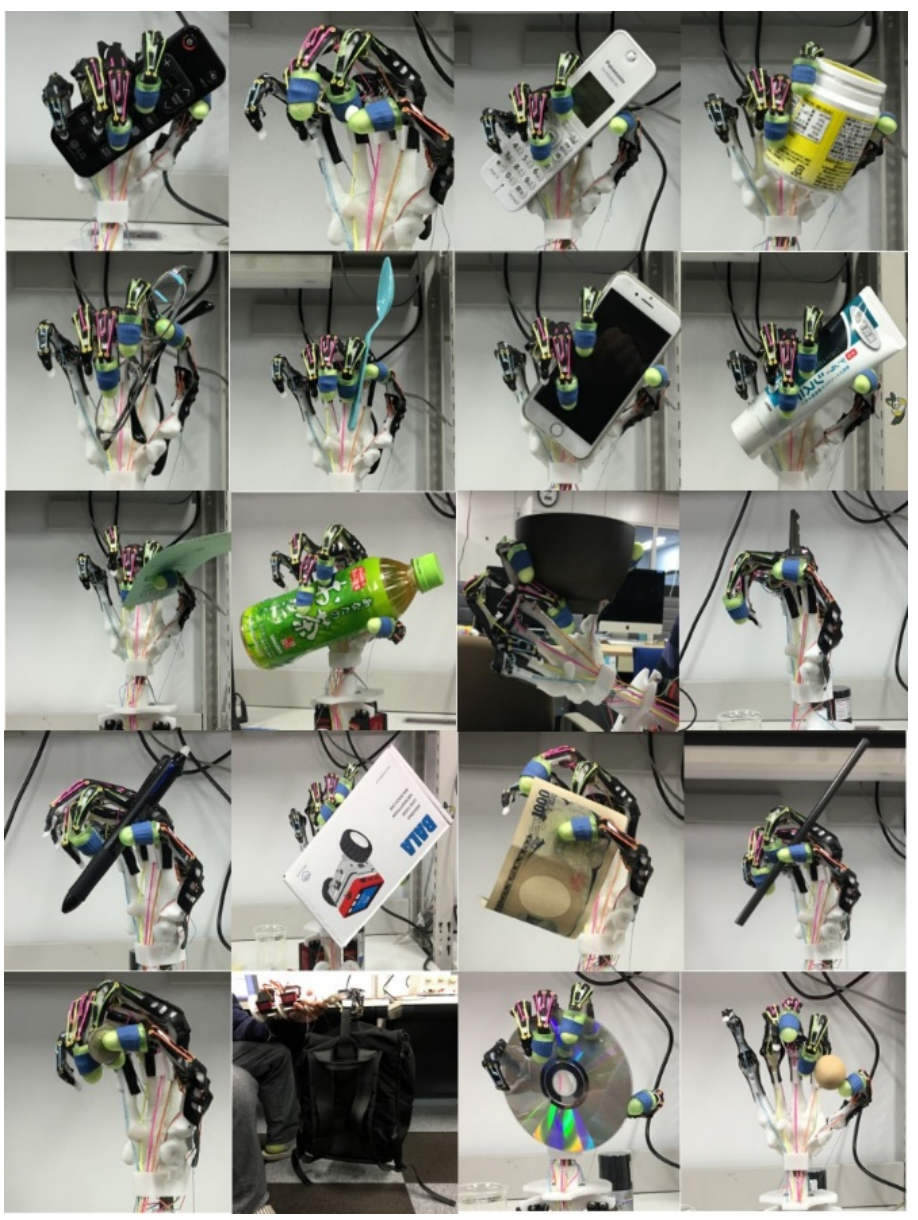

Figure 10: Result of grasping experiment

\subsection{Result of Classification experiment}

It showed the results of the movement Classification experiment in Table 4. The highest average accuracy rate was $93.7 \%$. The lowest average accuracy rate was $87.5 \%$. All subjects achieved 100\% accuracy when Classifying "Power grasp" Motion. In addition, the classification of "Precision grasp" and "Lateral grasp" is slightly lower than the accuracy rate of other motions, with a minimum of $80 \%$. During the experiment, the researchers observed that the subject's 'Relax' movements were classified as 'Lateral grasp' movements multiple times. This might because the

Table 4: Classification accuracy

\begin{tabular}{|c|c|c|c|c|}
\hline Motion & $\mathbf{1}$ & $\mathbf{2}$ & $\mathbf{3}$ & $\mathbf{4}$ \\
\hline \multirow{2}{*}{ Power grasp } & $\begin{array}{c}20 / 20 \\
100 \%\end{array}$ & $\begin{array}{c}20 / 20 \\
100 \%\end{array}$ & $\begin{array}{c}20 / 20 \\
100 \%\end{array}$ & $\begin{array}{c}20 / 20 \\
100 \%\end{array}$ \\
\hline Precision & $18 / 20$ & $18 / 20$ & $17 / 20$ & $16 / 20$ \\
grasp & $90 \%$ & $90 \%$ & $85 \%$ & $80 \%$ \\
\hline \multirow{2}{*}{ Lateral grasp } & $20 / 20$ & $16 / 20$ & $18 / 20$ & $17 / 20$ \\
& $100 \%$ & $80 \%$ & $90 \%$ & $85 \%$ \\
\hline Relax & $17 / 20$ & $19 / 20$ & $18 / 20$ & $17 / 20$ \\
& $85 \%$ & $95 \%$ & $90 \%$ & $85 \%$ \\
\hline AVG. & $93.7 \%$ & $91.2 \%$ & $91.2 \%$ & $87.5 \%$ \\
\hline
\end{tabular}

EMG signal generated by thumb adduction is difficult to collect from superficial muscles.

\section{Discussion}

In the Grasping experiment, we tested the gripping performance of the proposed prosthesis against objects commonly found in daily environments. When grasping objects such as cylindrical objects or large objects, we usually use the Power grasp posture. The $500 \mathrm{ml}$ PET bottle and the $18 \times 11 \times 8 \mathrm{~cm}$ paper bag are very large for the hand, so it is necessary to grasp them with your fingertips. In addition, the artificial hand can lift a bag of about 1 $\mathrm{kg}$ and hold the posture for over 5 seconds. From the above results, it can be found that the anatomically reproduced finger structure sufficiently contributes to the transmission of force and transmits the torque of the motor to the fingers and fingertips. Although a strong torque moment applies to the fingertips, the shape of the finger joints is not deformed, and the proposed artificial hand has sufficient force and rigidity to grasp heavy objects. By using precision grasp and Lateral grasp, the prosthetic hand can pick up small objects such as balls and coins with fingertips during the experiment.

These results shown that our proposed design shows highly coordinated motions of the thumb and index finger, suggested that the proposed thumb structure has the same kinetic features as a human thumb. It is also one of our design goals to use bones as a model to give the artificial hand a natural appearance. As shown in Figure 11, our prosthetic hand also holds Natural-Appearance when wearing a silicon skin glove. It should be noted that the silicon skin glove worn is only a general commercial product. 
For healthy subjects, the ANN classification system can achieve high-precision classification of 4 motions. But before the system is used in amputees, it is still necessary to continue to improve performance. From the experimental results, the accuracy of the two motions of "precise grasp" and "side grasp" is lower than other motions. The reason may be that "precise grip" and "side grip" include thumb movement, so the feature sets of these two motions are similar. In our experiment, the EMG signal is measured by two EMG sensors in the flexor group and two EMG sensors in the extensor group. However, it may be difficult to classify detailed finger movements from the extracted EMG signals. Because there are many muscles in the forearm (Flexor carpi radialis, flexor carpi ulnar is, Flexor digitorum superficialis muscle, Deep digital flexor muscle, flexor digitorum longus) located deep in the measurement position. This will affect the accuracy and precision of classification.

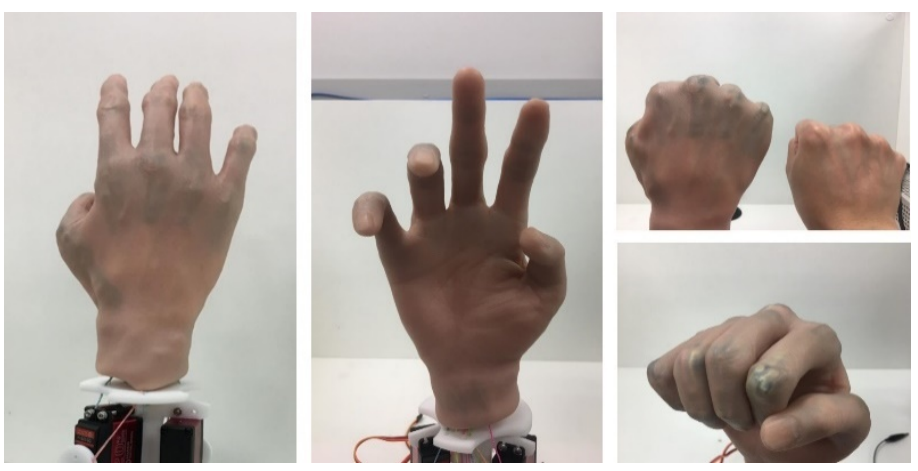

Figure 11: Appearance of prosthetic hand wearing silicon skin glove

\section{Conclusion}

In this study, we developed a biomimetic prosthetic hand based on human anatomy. By reproducing critical soft tissues such as tendons and ligaments in the human hand, the developed artificial hand has the same dexterity as a human finger. For this reason, our prosthetic hand can stably grasp all the object types proposed in the experiment. In order to control the prosthetic hand by EMG signal, an ANN has been designed for classifying four motions. Four subjects tested the classification performance of ANN. The classification accuracy average rate was $91 \%$.

The limitations of this study are as follows. The overall system of the proposed bionic prosthesis is about 560 grams. A survey of prosthetic users pointed out that if the weight of the prosthetic hand exceeds 400 grams, and it is considered being heavy [34]. Currently, our biomimetic prosthesis does not include the wrist part. In order to use artificial hands in daily life, the ability of the wrist part also plays a vital role. Currently, the proposed EMG control method only tests the performance of healthy subjects, and needs to be tested on amputees to investigate whether the performance will change. In the next phase of this research, we will make improvements to address these issues.

\section{Conflict of Interest}

The authors declare no conflict of interest.

\section{Acknowledgment}

This work was supported by JSPS KAKENHI Grant Number 19K11428.

\section{References}

[1] Z. He, R.R. Yurievich, S. Shimizu, M. Fukuda, Y. Kang, D. Shin, “A Design of Anthropomorphic Hand based on Human Finger Anatomy," in 2020 International Symposium on Community-centric Systems (CcS), IEEE: 1-5, 2020, doi:10.1109/CcS49175.2020.9231423.

[2] K. Ziegler-Graham, E.J. MacKenzie, P.L. Ephraim, T.G. Travison, R. Brookmeyer, "Estimating the Prevalence of Limb Loss in the United States: 2005 to 2050," Archives of Physical Medicine and Rehabilitation, 89(3), 422-429, 2008, doi:10.1016/J.APMR.2007.11.005.

[3] F. Cordella, A.L. Ciancio, R. Sacchetti, A. Davalli, A.G. Cutti, E. Guglielmelli, L. Zollo, "Literature Review on Needs of Upper Limb Prosthesis Users," Frontiers in Neuroscience, 0(MAY), 209, 2016, doi:10.3389/FNINS.2016.00209.

[4] E.A. Biddiss, T.T. Chau, "Upper limb prosthesis use and abandonment: A survey of the last 25 years," doi:10.1080/03093640600994581.

[5] I. Vujaklija, D. Farina, O.C. Aszmann, "New developments in prosthetic arm systems," Orthopedic Research and Reviews, 8, 31, 2016, doi:10.2147/ORR.S71468.

[6] G. Langevin. InMoov - open-source 3D printed life-size robot, http://inmoov.fr/, Aug. 2021

[7] Exiii Inc. HACKberry |3D-printable open-source bionic arm, http://exiiihackberry.com/, Aug. 2021.

[8] J. Zuniga, D. Katsavelis, J. Peck, J. Stollberg, M. Petrykowski, A. Carson, C. Fernandez, "Cyborg beast: a low-cost 3d-printed prosthetic hand for children with upper-limb differences," BMC Research Notes 2015 8:1, 8(1), 1-9, 2015, doi:10.1186/S13104-015-0971-9.

[9] P. Weiner, J. Starke, F. Hundhausen, J. Beil, T. Asfour, "The KIT Prosthetic Hand: Design and Control," IEEE International Conference on Intelligent Robots and Systems, 3328-3334, 2018, doi:10.1109/IROS.2018.8593851.

[10] P. Weiner, C. Neef, Y. Shibata, Y. Nakamura, T. Asfour, "An Embedded, Multi-Modal Sensor System for Scalable Robotic and Prosthetic Hand Fingers," Sensors 2020, Vol. 20, Page 101, 20(1), 101, 2019, doi:10.3390/S20010101.

[11] S. Lee, S. Noh, Y.K. Lee, J.H. Park, "Development of bio-mimetic robot hand using parallel mechanisms," 2009 IEEE International Conference on Robotics and Biomimetics, ROBIO 2009, 550-555, 2009, doi:10.1109/ROBIO.2009.5420706.

[12] A. Mohammadi, J. Lavranos, H. Zhou, R. Mutlu, G. Alici, Y. Tan, P. Choong, D. Oetomo, "A practical 3D-printed soft robotic prosthetic hand with multiarticulating capabilities," PLOS ONE, 15(5), e0232766, 2020, doi:10.1371/JOURNAL.PONE.0232766.

[13] A.D. Deshpande, Z. Xu, M.J.V. Weghe, B.H. Brown, J. Ko, L.Y. Chang, D.D. Wilkinson, S.M. Bidic, Y. Matsuoka, "Mechanisms of the anatomically correct testbed hand," IEEE/ASME Transactions on Mechatronics, 18(1), 238-250, 2013, doi:10.1109/TMECH.2011.2166801.

[14] Z. Xu, E. Todorov, "Design of a highly biomimetic anthropomorphic robotic hand towards artificial limb regeneration," Proceedings - IEEE International Conference on Robotics and Automation, 2016-June, 3485-3492, 2016, doi:10.1109/ICRA.2016.7487528.

[15] M.B.I. Reaz, M.S. Hussain, F. Mohd-Yasin, M.B.I. Raez, "Techniques of EMG signal analysis: detection, processing, classification and applications," Biol. Proced. Online, 8(1), 11-35, 2006, doi:10.1251/bpol15.

[16] O. Faust, Y. Hagiwara, T.J. Hong, O.S. Lih, U.R. Acharya, "Deep learning for healthcare applications based on physiological signals: A review," Computer Methods and Programs in Biomedicine, 161, 1-13, 2018, doi:10.1016/J.CMPB.2018.04.005.

[17] A. Kalantari, A. Kamsin, S. Shamshirband, A. Gani, H. Alinejad-Rokny, A.T. Chronopoulos, "Computational intelligence approaches for classification of medical data: State-of-the-art, future challenges and research directions," Neurocomputing, 276, 2-22, 2018, doi:10.1016/J.NEUCOM.2017.01.126.

[18] U. Côtéallard, F. Nougarou, C.L. Fall, P. Gigu'ere, C. Gosselin, F. Laviolette, B. Gosselin, "A Convolutional Neural Network for robotic arm guidance using sEMG based frequency-features," IEEE International Conference on Intelligent Robots and Systems, 2016-November, 2464-2470, 2016, doi:10.1109/IROS.2016.7759384.

[19] M. Tavakoli, C. Benussi, P. Alhais Lopes, L.B. Osorio, A.T. de Almeida, "Robust hand gesture recognition with a double channel surface EMG wearable armband and SVM classifier," Biomedical Signal Processing and Control, 46, 121-130, 2018, doi:10.1016/J.BSPC.2018.07.010.

[20] I.M. Bullock, J.Z. Zheng, S. De La Rosa, C. Guertler, A.M. Dollar, "Grasp frequency and usage in daily household and machine shop tasks," IEEE Transactions on Haptics, 6(3), 296-308, 2013, doi:10.1109/TOH.2013.6. 
[21] C.C. Scuola, S. Sant'anna, M.C. Scuola, M. Chiara, C. Scuola, C. Cipriani, M. Controzzi, M. Chiara Carrozza, "Objectives, criteria and methods for the design of the SmartHand transradial prosthesis Sensory feedback and closed loop control of artificial limbs View project Artificial arms components View project Objectives, criteria and methods for the design of the SmartHand transradial prosthesis," Robotica, 28, 919-927, 2010, doi:10.1017/S0263574709990750.

[22] C.L. Taylor, R.J. Schwarz, "The Anatomy and Mechanics of the Human Hand," Artificial Limbs VOL. 2 MAY 1955 NO. 2 CONTENTS, 2, 1955.

[23] Human Hand Bones - Thumb by siderits - Thingiverse, https://www.thingiverse.com/thing:15342, Aug. 2021.

[24] J.C. Becker, N. V. Thakor, "A Study of the Range of Motion of Human Fingers with Application to Anthropomorphic Designs," IEEE Transactions on Biomedical Engineering, 35(2), 110-117, 1988, doi:10.1109/10.1348.

[25] I. Komatsu, J.D. Lubahn, "Anatomy and Biomechanics of the Thumb Carpometacarpal Joint," Operative Techniques in Orthopaedics, 28(1), 1-5, 2018, doi:10.1053/J.OTO.2017.12.002.

[26] EDUCATION EXHIBIT Extensor Mechanism of the Fingers: MR ImagingAnatomic Correlation 1, Aug. 2021, doi:10.1148/rg.233025079.

[27] A. Gailey, P. Artemiadis, M. Santello, "Proof of Concept of an Online EMGBased Decoding of Hand Postures and Individual Digit Forces for Prosthetic Hand Control," Frontiers in Neurology, 0(FEB), 7, 2017, doi:10.3389/FNEUR.2017.00007.

[28] MathWorks Instrument Control Toolbox Team (2021). BITalino Toolbox, https://www.mathworks.com/matlabcentral/fileexchange/53983-bitalinotoolbox, Aug. 2021.

[29] Y. Koike, M. Kawato, "Estimation of dynamic joint torques and trajectory formation from surface electromyography signals using a neural network model," Biological Cybernetics 1995 73:4, 73(4), 291-300, 1995, doi:10.1007/BF00199465.

[30] A. Phinyomark, C. Limsakul, P. Phukpattaranont, "A Novel Feature Extraction for Robust EMG Pattern Recognition," Journal of Medical Engineering and Technology, 40(4), 149-154, 2009.

[31] W. Chen, M. Liu, S. Yue, W. Chen, C.-H. Xiong, W.-R. Chen, B.-Y. Sun, M.-J. Liu, S.-G. Yue, W.-B. Chen, "Design and Implementation of an Anthropomorphic Hand for Replicating Human Grasping Functions," IEEE TRANSACTIONS ON ROBOTICS, 32(3), 2016, doi:10.1109/TRO.2016.2558193.

[32] A.R. Siddiqi, S.N. Sidek, "Estimation of continuous thumb angle and force using electromyogram classification," Aug. 2021, doi: $10.1177 / 1729881416658179$.

[33] S.C. Young, T. Deyle, T. Chen, J.D. Glass, C.C. Kemp, "A list of household objects for robotic retrieval prioritized by people with ALS," 2009 IEEE International Conference on Rehabilitation Robotics, ICORR 2009, 510-517, 2009, doi:10.1109/ICORR.2009.5209484.

[34] J.T. Belter, A.M. Dollar, "Performance characteristics of anthropomorphic prosthetic hands," IEEE International Conference on Rehabilitation Robotics, 2011, doi:10.1109/ICORR.2011.5975476. 